\title{
Theoretical Studies on Electrophilic Aromatic Substitution Reaction for 8-Hydroxyquinoline
}

\author{
HASAN R. OBAYES ${ }^{1}$, KHALIDA F. AL.AZAWI ${ }^{1}$, \\ SHAYMAA H. KHAZAAL ${ }^{1}$, GHADAH H. ALWAN ${ }^{2}$, ABDULNASSER M. AL-GEBORI', \\ ALI H. AL-HAMADANI ${ }^{3}$ and AHMED AL-AMIERY ${ }^{3 *}$
}

${ }^{1}$ Applied Chemistry Division, Applied Science Department, University of Technology, Baghdad, Iraq. ${ }^{2}$ Ministry of Sciences and Technology, Industrial Research \& Development Directorate, Industrial Applications Centre, Baghdad, Iraq.

${ }^{3}$ Energy and Renewable Energies Technology Centre,

University of Technology (UOT), Baghdad 10001, Iraq.

*Corresponding author E-mail: dr.ahmed1975@gmail.com

http://dx.doi.org/10.13005/ojc/320127

(Received: December 26, 2015; Accepted: January 31, 2016)

\begin{abstract}
Theoretical investigations of organic molecules for the objective of their structural stability are the most important techniques in this regards. Recently calculations and simulation reactions utilizing theoretical studies become attractive conventional method for the researchers. Density function theory (DFT) method was used to study the reaction of 8-hydroxyquinoline with 4-ethoxycarbonyl-benzene diazonium chloride as electrophilic aromatic substitution reaction. To study any reaction there are two explanations: first explanation depends on the reactant molecules and second explanation depends on the stability of the product molecules. Determine the stability of the molecule by comparing the energies (total energy, energy level of (HOMO), and energy gap), we have three stable molecules, are: HQ-7-YBAEE (II) for the total energy, HQ-6-YBAEE (II) for the energy level of (HOMO) and $\mathrm{HQ}-2$-YBAEE (II) for the energy gap. The molecule HQ-4-YBAEE (II) is always at least stability in all data.
\end{abstract}

Key words: Electrophilic, 8-Hydroxyquinoline, DFT, HOMO, Energy gap, Total energy.

\section{INTRODUCTION}

Theoretical studies had been quite used to investigate the reaction mechanism, explain the reaction products and clarify chemical reactions mystery. Theoretical studies are valuable approaches to explore the mechanism of reactions in the molecules and their electronic structures levels in addition to electronic parameters that acquired by means of theoretical calculations employ the computational methods of quantum chemistry ${ }^{1,2}$. The improvement in theoretical 
studies and applications have accomplish a point where predicted features of logical accuracy can be obtained from DFT (density functional theory) studies ${ }^{3,4}$. Geometry of the molecules in its ground state, as well as the nature of their molecular orbitals, highest occupied molecular orbital (HOMO) and lowest unoccupied molecular orbital (LUMO) are involved in the properties of activity of molecules reaction. Density Function Theory (DFT), a DFT calculation adds an additional step to each major phase of a Hartree-Fock calculation. This step is a numerical integration of the functional (or various derivatives of the functional) ${ }^{5}$. Thus in addition to the sources of numerical error in Hartree-Fock calculations (integral accuracy, SCF convergence, and CPHF convergence), the accuracy of DFT calculations also depends on number of points used in the numerical integration. The "fine" integration grid is the default in Gaussian 09. This grid greatly enhances calculation accuracy at minimal additional $\operatorname{cost}^{6}$. We do not recommend using any smaller grid in production DFT calculations. Note also that it is important to use the same grid for all calculations where you intend to compare energies (e.g., computing energy differences, heats of formation, and so on). Larger grids are available when needed (e.g. tight optimization of certain kinds of systems). An alternate grid may be selected by including in the route section ${ }^{7}$. The B BLYP density functional theory calculations exhibit good performance on the molecular geometry and vibrational properties of organic compounds ${ }^{8,9}$. To extend our studies on the design and synthesis of new compounds ${ }^{10-22}$, we describe here, investigate the dependence of stability of the molecules on theoretical chemical parameters such as total energy, energy level of (HOMO), and energy gap. We have three stable molecules, HQ-7YBAEE (II) for the total energy, HQ-6-YBAEE (II) for the energy level of (HOMO) and HQ-2-YBAEE (II) for the energy gap. The molecule HQ-4-YBAEE (II) is always at least stability in all data.

\section{The Calculation Method}

To calculate ground-state geometries, Gaussian 09, revision A.02 ${ }^{23}$ was optimized to a local minimum without symmetry restrictions using basis set $6-31 \mathrm{G}^{24,25}$. The combination of the Becke three-parameter hybrid (B3) ${ }^{26}$ exchange functional and the Lee-Yang-Parr (LYP) (Lee, Yang, Parr, 1988correlation functional (B3LYP) ${ }^{27,28}$, was used for all geometry optimizations, Highest Occupied Molecular Orbital Energies $\left(\mathrm{E}_{\text {номо }}\right)$, Lowest Unoccupied Molecular Orbital Energies ( $\mathrm{E}_{\text {Lumo }}$ ), and physical properties for the molecules in this study.

\section{RESULTS AND DISCUSSION}

The reaction 8-hydroxyquinoline with 4-ethoxycarbonyl-benzene diazonium chloride described below as in Scheme 1, was selected for study the electrophilic aromatic substitution reaction theoretically by using density function theory (DFT).

The molecule 8-hydroxyquinoline electrophilic aromatic substitution reaction theoretically has six possibilities due to the presence of six hydrogen on aromatic carbon atoms which is associated with the C-2 to C-7, for the purpose of discussing the possibilities of this reaction there are two explanations: first explanation depends on the reactant molecules or call the name (explanation of the pre-reaction), which now has a lot of research, the second explanation depends on the stability of the product molecules, which we will focus on in this work ${ }^{29-33}$.

\section{Explanation of the pre-reaction}

Figure 1. shows the electronic distribution of the molecule 8-hydroxyquinoline when the energy level of highest occupied molecular orbital (HOMO) and lowest unoccupied molecular orbital (LUMO), if we look to the electronic distribution of $\mathrm{HOMO}$ note electron density high for each of the carbon atoms C-2 to C-6, which means the probability equal to a substitution reaction, making it difficult to determine directing substitution on site given alone.

\section{Explanation of the post-reaction}

This explanation depended on the stability of the product molecules; first, you must know the number of the product molecules and second to study of these molecules through find the total energy and the energy level of (HOMO), the number of the product molecules are twelve are shown in Figure 2. and Figure 3. use symbols by name because of the length of the molecule name, 4-ethoxycarbonylbenzene diazonium is attached to an aromatic system (usually hydrogen) is replaced by an electrophile, six hydrogens will be replaced; so we will have six 


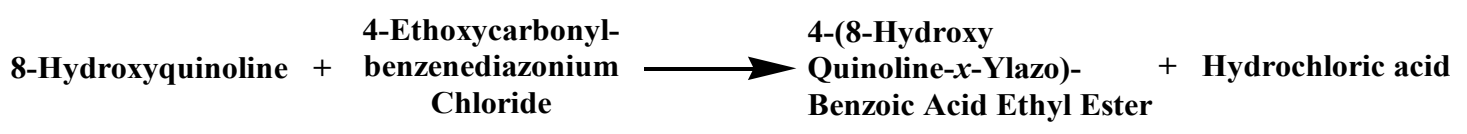<smiles>[2H]c1cccc2cccnc12</smiles>

8-Hydroxyquinoline

Q

Benzoic Acid Ethyl Ester

$$
x=2,3,4,5,6,7
$$

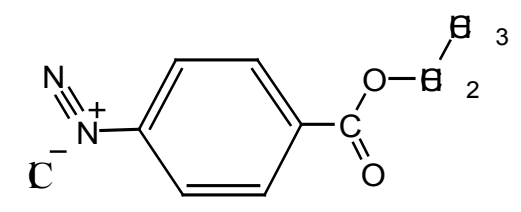

4-Ethoxycarbonyl-benzenediazonium Chloride

Scheme 1: Synthesis of the target compound

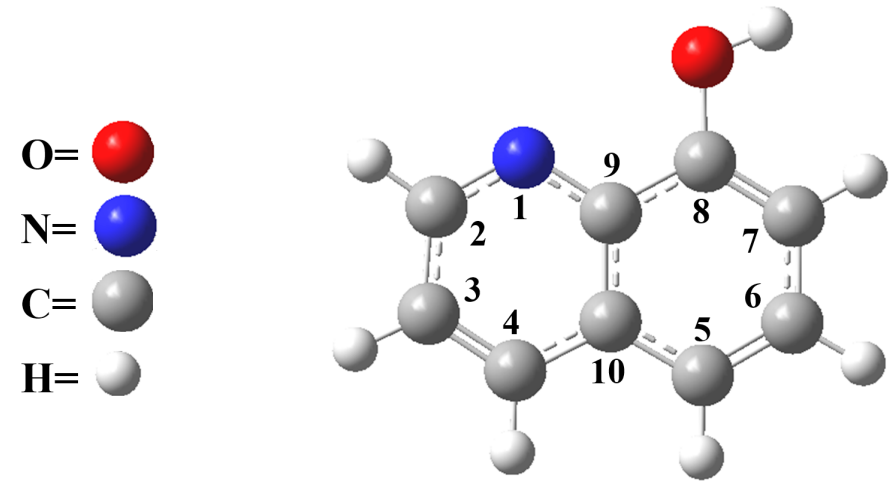

8-Hydroxyquinoline

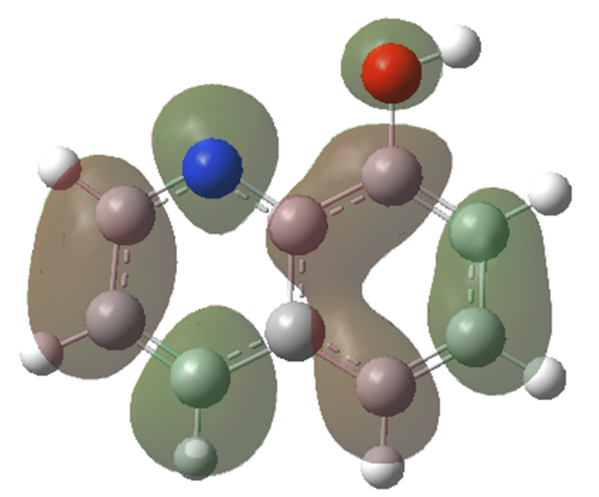

HOMO

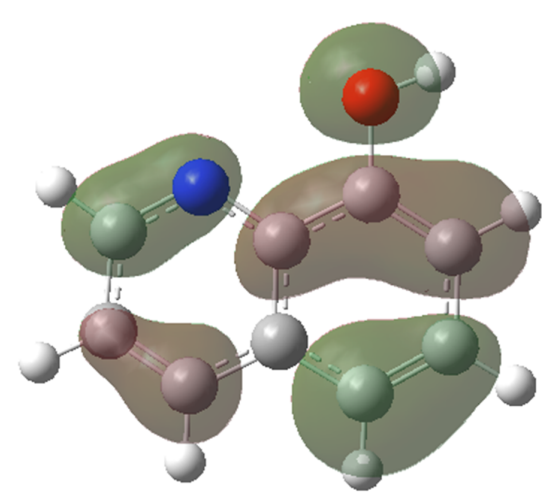

LUMO

Fig. 1: The distribution of electron density of (HOMO) and (LUMO) for 8-hydroxyquinoline molecule by using RB3LYP/6-31G method 


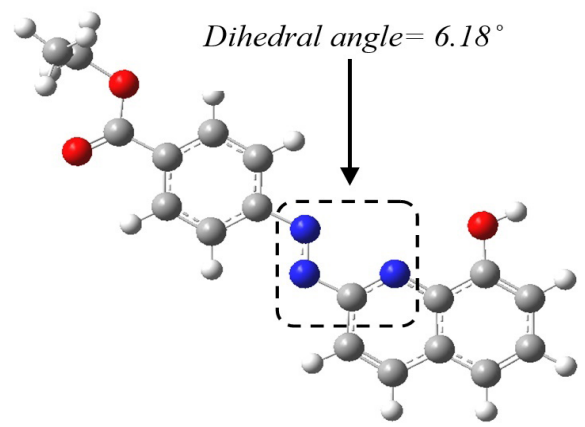

HQ-2-YBAEE (I)

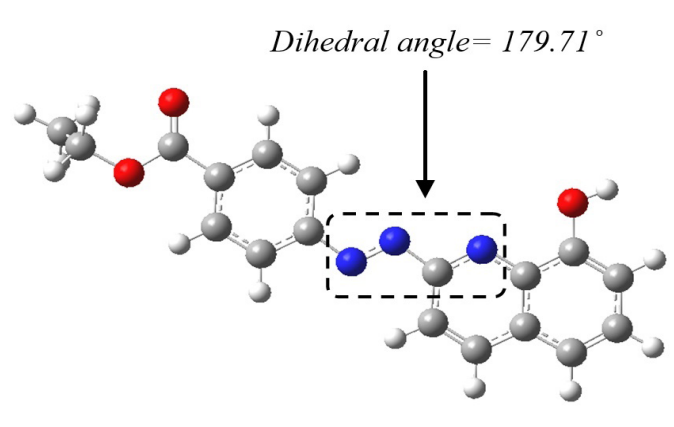

HQ-2-YBAEE (II)

4-(8-Hydroxy Quinoline-2-Ylazo)-Benzoic Acid Ethyl Ester

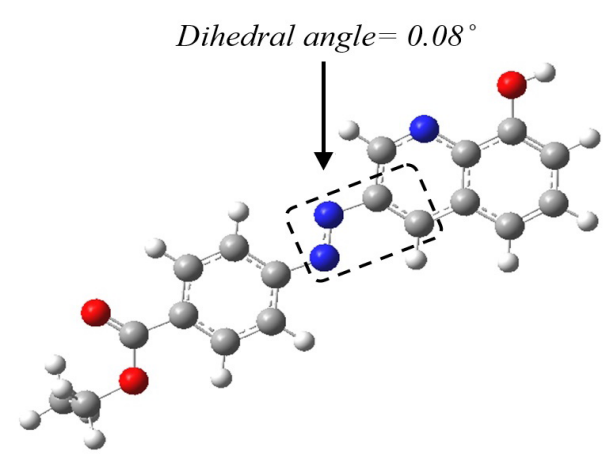

HQ-3-YBAEE (I)

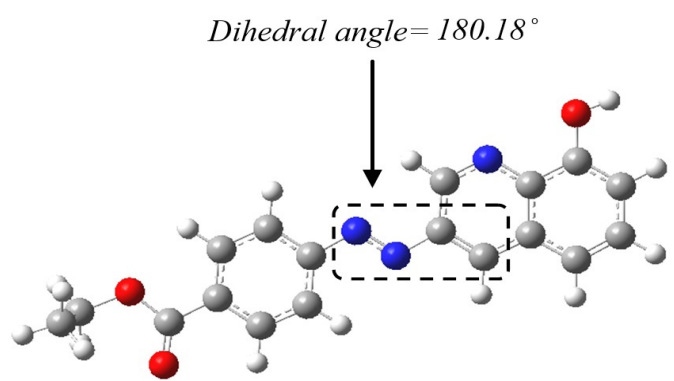

HQ-3-YBAEE (II)

4-(8-Hydroxy Quinoline-3-Ylazo)-Benzoic Acid Ethyl Ester

Dihedral angle $=1.10^{\circ}$

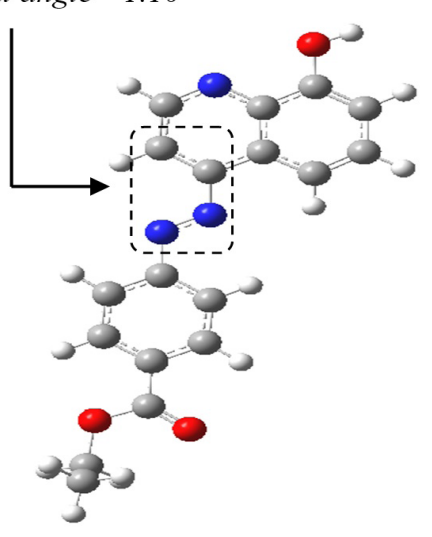

HQ-4-YBAEE (I)
Dihedral angle $=180.70^{\circ}$

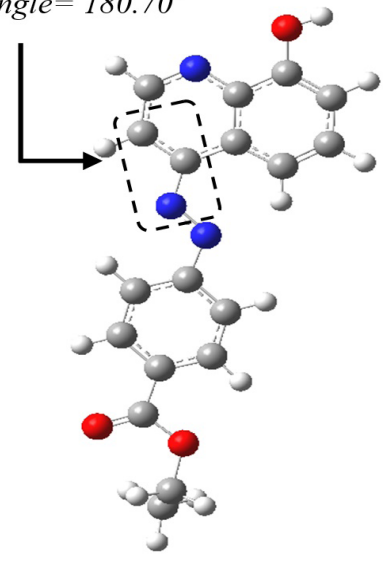

HQ-4-YBAEE (II)

4-(8-Hydroxy Quinoline-4-Ylazo)-Benzoic Acid Ethyl Ester

Fig. 2: The optimized structure of HQ-2-YBAEE, HQ-3-YBAEE and HQ-4-YBAEE molecule by using RB3LYP/6-31G method 


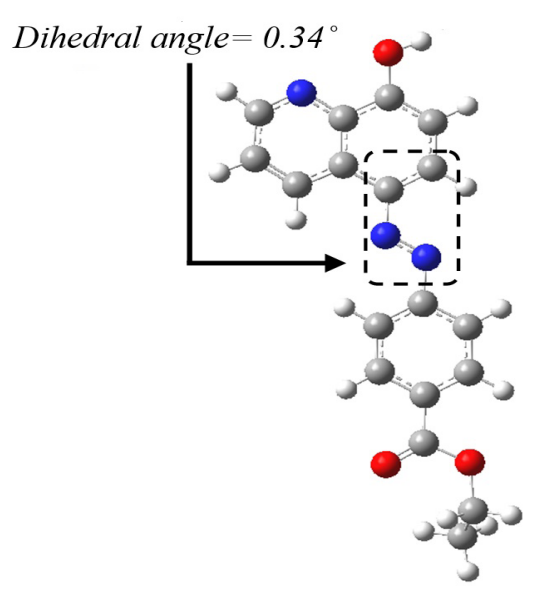

HQ-5-YBAEE (I)

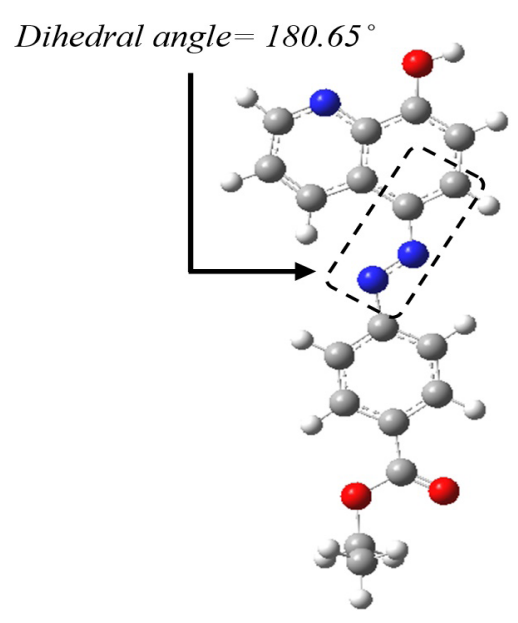

HQ-5-YBAEE (II)

4-(8-Hydroxy Quinoline-5-Ylazo)-Benzoic Acid Ethyl Ester

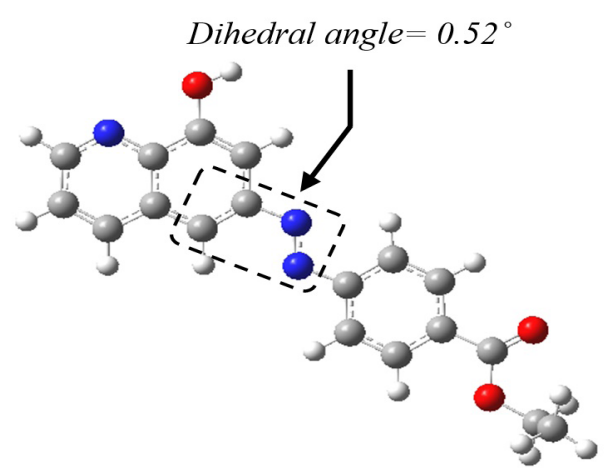

HQ-6-YBAEE (I)

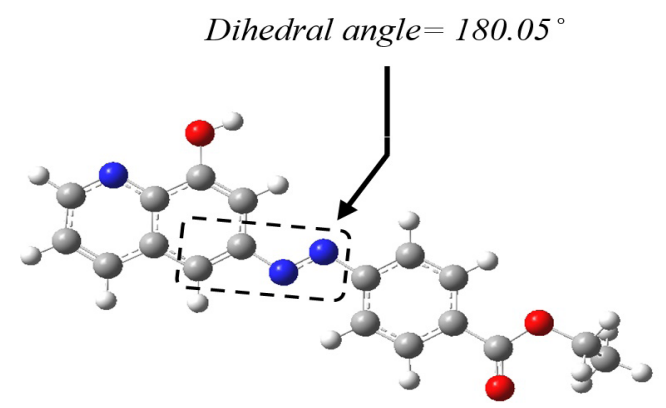

HQ-6-YBAEE (II)

4-(8-Hydroxy Quinoline-6-Ylazo)-Benzoic Acid Ethyl Ester

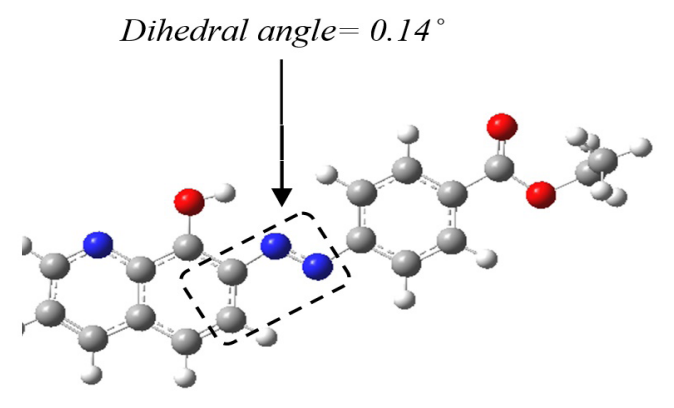

HQ-7-YBAEE (I)

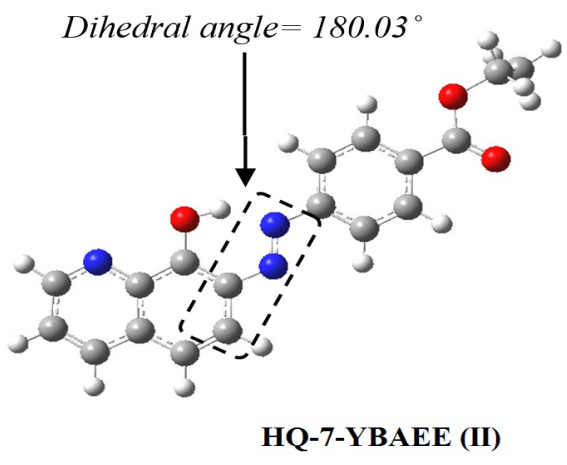

HQ-7-YBAEE (II)

4-(8-Hydroxy Quinoline-7-Ylazo)-Benzoic Acid Ethyl Ester

Fig. 3: The optimized structure of HQ-5-YBAEE, HQ-6-YBAEE and HQ-7-YBAEE molecule by using RB3LYP/6-31G method 
Table 1: Some energies value for all molecules were calculated in B3LYP/6-31G method

\begin{tabular}{|c|c|c|c|c|}
\hline Molecules & Total Energya.u. & $\mathrm{E}_{\text {номо }} \mathrm{eV}$ & $\mathrm{E}_{\text {Lumo }} \mathrm{eV}$ & $\begin{array}{c}\text { Gap energy } \\
\left(E_{\text {Lumo }}-E_{\text {номо }}\right) e V\end{array}$ \\
\hline 8-Hydroxyquinoline & -477.01192 & -5.8502 & -1.3021 & 4.5481 \\
\hline $\begin{array}{l}\text { 4-Ethoxycarbonyl- } \\
\text { benzenediazonium } \\
\text { Chloride }\end{array}$ & -1068.29242 & -7.0116 & -3.4240 & 3.5876 \\
\hline HQ-2-YBAEE (I) & -1084.52958 & -5.9539 & -2.7217 & 3.2322 \\
\hline HQ-2-YBAEE (II) & -1084.53374 & -6.0118 & -2.7434 & 3.2684 \\
\hline HQ-3-YBAEE (I) & -1084.53280 & -6.0325 & -2.9010 & 3.1315 \\
\hline HQ-3-YBAEE (II) & -1084.53312 & -6.0545 & -2.8098 & 3.2447 \\
\hline HQ-4-YBAEE (I) & -1084.53192 & -5.8994 & -3.0482 & 2.8512 \\
\hline HQ-4-YBAEE (II) & -1084.52857 & -5.9530 & -2.9875 & 2.9655 \\
\hline HQ-5-YBAEE (I) & -1084.53382 & -5.9125 & -2.7587 & 3.1538 \\
\hline HQ-5-YBAEE (II) & -1084.53656 & -5.9732 & -2.7075 & 3.2657 \\
\hline HQ-6-YBAEE (I) & -1084.53342 & -6.0126 & -2.8648 & 3.1478 \\
\hline HQ-6-YBAEE (II) & -1084.53581 & -6.0730 & -2.8128 & 3.2602 \\
\hline HQ-7-YBAEE (I) & -1084.54421 & -5.9963 & -2.8760 & 3.1203 \\
\hline HQ-7-YBAEE (II) & -1084.55187 & -5.9998 & -2.9437 & 3.0561 \\
\hline
\end{tabular}

products of each two-isomer as a result of change dihedral angle because of the steric affect.

All twelve molecules were studied using the method of calculation (RB3LYP/6-31G) and the results found in the table 1 . We note that the second isomer (II) when the value of dihedral angle to be about 180 degrees is more stable than first isomer (I) when the value of dihedral angle to be about zero degrees due to decrease repulsion energy because of the steric affect, thus been reduced possible molecules to six molecules are [HQ-2-YBAEE (II), HQ-3-YBAEE (II), HQ-4-YBAEE (II), HQ-5-YBAEE (II), HQ-6-YBAEE (II), and HQ-7-YBAEE (II)]. Of the total energy and the energy level of (HOMO) can find the most stability of the molecule and thus can be expected to substitution on any site is a high percentage, molecule HQ-7-YBAEE (II) is more stable than the rest of the other molecules based on the total energy have equal value (-1084.55187 au), can rearrangement of the molecules in descending order for the decrease stability, also at the bottom.

HQ-7-YBAEE (II) > HQ-5-YBAEE (II) > HQ-6-YBAEE (II) > HQ-2-YBAEE (II) > HQ-3-YBAEE (II) > HQ-4-YBAEE (II)
While for energy level of (HOMO), molecule HQ-6-YBAEE (II) is more stable than the rest of the other molecules based on energy level of (HOMO) have equal value $(-6.0730 \mathrm{eV})$, can rearrangement of the molecules in descending order for the decrease stability, also at the bottom.

$$
\begin{aligned}
& \text { HQ-6-YBAEE (II) > HQ-3-YBAEE (II) > HQ-2-YBAEE (II) > } \\
& \text { HQ-7-YBAEE (II) > HQ-5-YBAEE (II) > HQ-4-YBAEE (II) }
\end{aligned}
$$

Energy gap; the energy gap is also called band gap, the gap energy generally refers to the energy difference (in electron volts) between the Low Unoccupied Molecular Orbital (LUMO) and the High Occupied Molecular Orbital (HOMO) in insulators and semiconductors. This is equivalent to the energy required to free an outer shell electron from its orbit about the nucleus to become a mobile charge carrier, therefore, the molecule is stable, which has a large energy gap, molecule HQ-2-YBAEE (II) is more stable than the rest of the other molecules based on energy gap have equal value (3.2684 eV), can rearrangement of the molecules in descending order for the decrease stability, also at the bottom.

HQ-2-YBAEE $($ II) > HQ-5-YBAEE $($ II) $>$ HQ-6-YBAEE $($ II) $>$ HQ-3-YBAEE (II) > HQ-7-YBAEE (II) > HQ-4-YBAEE (II) 
So we have three stable molecules, are: HQ-7-YBAEE (II) for the total energy, HQ-6-YBAEE (II) for the energy level of (HOMO), HQ-2-YBAEE (II) for the energy gap. The molecule HQ-4-YBAEE (II) is always at least stability in all data.

\section{CONCLUSION}

A quantum chemistry calculation is carried out using the density function theory (DFT) method to study optimized for twelve molecules, to study the electrophilic aromatic substitution reaction using two explanations, first explanation depends on the reactant molecules and second explanation depends on the product molecules. We have three stable molecules, are: HQ-7-YBAEE (II) for the total energy, HQ-6-YBAEE (II) for the energy level of (HOMO), HQ-2-YBAEE (II) for the energy gap. The molecule HQ-4-YBAEE (II) is always at least stability in all data.

\section{REFERENCES}

1. Zhou, M. Li, Y. and, Dang, L. Theoretical Studies on the Mechanism of the Michael Addition Reaction Catalyzed by a ThioureaCinchona-Amine: Triple Activation. Asian Journal of Organic Chemistry, 2015, 4, 904-911

2. Radenkoviæ, S. Tošoviæ, J. Havenith, R. Bultinck, P. Ring Currents in Benzo- and Benzocyclobutadieno-Annelated Biphenylene Derivatives. Chem. Phys. Chem., 2015, 16, 216-222

3. Güne ${ }^{\circ}$, E. Parlak, C. DFT, FT-Raman and FT-IR investigations of 5-methoxysalicylic acid, SpectrochimicaActa Part A: Molecular and Biomolecular Spectroscopy, 2011, 82, 504-514

4. Lee, C., Yang, W., \& Parr, R. G. Development of the Colle-Salvetti correlation-energy formula into a functional of the electron density, Physical Review B, 1988, 37, 785-789.

5. Johnson B. G. and Frisch, M. J. An implementation of analytic second derivatives of the gradient-corrected density functional energy, J. Chem. Phys., 1994, 100, 74297442.

6. Stratmann, R. E. Burant, J. C. Scuseria, G. E. and Frisch, M. J. Improving harmonic vibrational frequencies calculations in density functional theory, J. Chem. Phys., 1996, 106, 10175-10183.

7. Stephens, P. J. Devlin, F. J. Frisch, M. J. and Chabalowski, C. F. "Ab initio Calculation of Vibrational Absorption and Circular Dichroism Spectra Using Density Functional Force Fields, J. Phys. Chem., 1994, 98, 1162311627.

8. Prasad, M.V.S. Sri, N. U. Veeraiah, V. A combined experimental and theoretical studies on FT-IR, FT-Raman and UV-Vis spectra of 2-chloro-3-quinolinecarboxaldehyde. SpectrochimicaActa Part A: Molecular and Biomolecular Spectroscopy, 2015, 118, 163174.

9. Umar, Y. Density functional theory calculations of the internal rotations and vibrational spectra of 2-, 3- and 4-formyl pyridine. Spectrochimica Acta Part A: Molecular and Biomolecular Spectroscopy, 2009, 71, 1907-1913.

10. Al-Amiery A, Al-Majedy Y, Kadhum A, Mohamad A. New Coumarin Derivative as an Eco-Friendly Inhibitor of Corrosion of Mild Steel in Acid Medium. Molecules. 2015; 20; 366-383.

11. Kadhum A, Mohamad A, Hammed L, AlAmiery $A$, San N. Inhibition of mild steel corrosion in hydrochloric acid solution by new coumarin. Materials. 2014; 7: 4335-4348.

12. Mohamad A, Kadhum A, Al-Amiery A, Ying L, Musa A. Synergistic of a coumarin derivative with potassium iodide on the corrosion inhibition of aluminum alloy in 1.0 M H2SO4. Metals and Materials International. 2014; 20: 459-467.

13. Al-Amiery A, Kadhum A, Alobaidy A, Mohamad A, Hoon P. Novel corrosion inhibitor for mild steel in HCl. Materials. 2014; 7: 662672.

14. Al-Amiery A, Kadhum A, Mohamad A, Musa A, Li C. Electrochemical study on newly synthesized chlorocurcumin as an inhibitor for mild steel corrosion in hydrochloric acid. Materials. 2013; 6: 5466-5477.

15. Al-Amiery A, Al-Bayati R, Saour K, Radi M. Cytotoxicity, antioxidant, and antimicrobial 
activities of novel 2-quinolone derivatives derived from coumarin Res. Chem. Intermed. 2012; 38: 559-569.

16. Al-Amiery A, Musa A, Kadhum A, Mohamad A. The use of umbelliferone in the synthesis of new heterocyclic compounds. Molecules 2011, 16, 6833-6843.

17. Al-Amiery A, Musa A, Kadhum A, Mohamad A. The antioxidant activity of new coumarin derivatives. Int. J. Mol. Sci. 2011; 12: 57475761.

18. Kadhum A, Al-Amiery A, Shikara M, Mohamad A, Al-Bayati R. Synthesis, structure elucidation and DFT studies of new thiadiazoles. Int. J. Phys. Sci. 2012; 6: 6692-6697.

19. Al-Amiery A, Kadhum A, Mohamad A. Antifungal and antioxidant activities of pyrrolidone thiosemicarbazone complexes. Bioinorg. Chem. Appl. 2012; 2012: 1-7.

20. Al-AmieryA. Synthesis and antioxidant, antimicrobial evaluation, DFT studies of novel metal complexes derivate from Schiff base. Res. Chem. Intermed. 2012; 38: 745-759.

21. Al-Amiery A. Antimicrobial and antioxidant activities of new metal complexes derived from (E)-3-((5-phenyl-1, 3, 4-oxadiazol-2ylimino) methyl) naphthalen-2-ol.Med. Chem. Res. 2011; 21: 3204-3213.

22. Al-Amiery A, Kadhum A, Mohamad A. Antifungal activities of new coumarins. Molecules. 2012; 17: 5713-5723.

23. Frisch, M. J., Trucks, G. W., Schlegel, H. B., Scuseria, G. E., Robb, M. A., Cheeseman, J. R., ... Pople, J. A. Gaussian 03, Revision C.02. Wallingford, CT: Gaussian. 2004

24. Fujimoto, T., Suizu, R., Yoshikawa, H., \& Awaga, K. Molecular, crystal, and thin-film structures of octathio[8]circulene: release of antiaromatic molecular distortion and lamellar structure of self-assembling thin films. Chemistry-A European Journal, 2008, 14, 6053-6056.

25. Musa, A. Y., Ahmoda, W., Al-Amiery, A. A., Kadhum, A. H., \& Mohamad, A. B. Quantum chemical calculation for the inhibitory effect of compounds. Journal of Structural Chemistry 2013, 54 (2), 301-308.
26. Becke, A. D. Density-functional thermochemistry. III. The role of exact exchange. The Journal of Chemical Physics, 1993, 98, 5648-5652.

27. Obayes, H. R., Alwan, G. H., Al-Amiery, A. A., Kadhum, A. H., \& Mohamad, A. B. Thermodynamic and Theoretical Study of the Preparation of New Buckyballs from Corannulene, Coronene, and Circulene. Journal of Nanomaterials, 2013, 2013, 1-8.

28. Obayes, H. R., Alwan, G. H., Alobaidy, A. H., Al-Amiery, A. A., Kadhum, A. H., \& Mohamad, A. B. Quantum chemical assessment of benzimidazole derivatives as corrosion inhibitors. Chemistry Central Journal, 2014, 8, 1-8.

29. Al-Amiery, A. Obayes, H. Alwan, G. Kadhum, A and Mohamad. A. Synthesis and Theoretical Studies of Methyl 2-[(2-oxo-2H-chromen-4yl) oxy] acetate. Asian Journal of Chemistry, 2013, 25, 10357-10359.

30. Al-Amiery, A., Jaffar, H. D., Obayes, H. R., Musa, A. Y., Kadhum, A. H., \& Mohamad, A. Thermodynamic studies on 4-aminocoumarin tautomers. International Journal of Electrochemical Science, 2012, 7, 84688472. Retrieved from

31. Naama, J. H., Alwan, G. H., Obayes, H. R., Al-Amiery, A. A., Al-Temimi, A. H., \& Kadhum, A. B. Curcuminoids as antioxidants and theoretical study of stability of curcumin isomers in gaseous state. Research on Chemical Intermediates, 2013, 39, 40474059.

32. Obayes H.R., Al-Amiery A.A., Jaffar H.D., Musa A.Y., Kadhum A.H. and Mohamad A., Theoretical study for the preparation of sub-carbon nano tubes from the cyclic polymerization reaction of two molecules from corannulene, coronene and circulene aromatic compounds. J. Comput. Theor. Nanosci. 2013, 10, 2459-2463.

33. Obayes, H. R., Al Obaidy, A. Alwan, G. H., AlAmiery, Molecular simulation for novel carbon buckyball materials. Cogent Chemistry, 2015, 1, 1-8. 